\title{
Advances in periodic difference equations with open problems
}

\author{
Ziyad AlSharawi ${ }^{\dagger}$, Jose Cánovasł* Antonio Linero ${ }^{\S}$ \\ $\dagger$ Department of Mathematics and Statistics, Sultan Qaboos University, \\ P. O. Box 36, PC 123, Al-Khod, Sultanate of Oman \\ Email: alsha1zm@alsharawi.info \\ ‡Departamento de Matemática Aplicada y Estadística, \\ Universidad Politécnica de Cartagena, \\ Paseo de Alfonso XIII 30203, Cartagena, Murcia, Spain \\ Email: jose.canovas@upct.es \\ $\S$ Departamento de Matemáticas, Universidad de Murcia, \\ Campus de Espinardo, Murcia 30100, Spain \\ Email: lineroba@um.es
}

October 5, 2013

\begin{abstract}
In this paper, we review some recent results on the dynamics of semi-dynamical systems generated by the iteration of a periodic sequence of continuous maps. In particular, we state several open problems focused on the structure of periodic orbits, forcing between periodic orbits, sharing periodic orbits, folding and unfolding periodic systems, and on applications of periodic systems.
\end{abstract}

\section{Introduction}

Let $C(I)$ denote the set of continuous maps $f: I \rightarrow I$ where $I$ is a compact subinterval of the real line. We consider $f_{0}, \ldots, f_{p-1} \in C(I)$. These maps generate a semidynamical system [34], which we denote by $\left(I,\left[f_{0}, \ldots, f_{p-1}\right]\right)$. For any $x \in I$, the

${ }^{*}$ Corresponding author: jose.canovas@upct.es 
orbit through $x$ is denoted by $\operatorname{Orb}\left(x,\left[f_{0}, \ldots, f_{p-1}\right]\right)$ and given by the solution of the non-autonomous difference equation

$$
\left\{\begin{array}{l}
x_{0}=x, \\
x_{n+1}=f_{n \bmod p}\left(x_{n}\right) .
\end{array}\right.
$$

The number $p$ is called the period of the system and it is always considered to be minimal.

Periodic difference equations have been studied by several authors recently (see for instance $[6,8,9,11,15,16,24,29,30,31,34]$. The interest for studying periodic discrete systems is motivated by applications in population dynamics (see e.g. [28] and [40]) and economic dynamics (see the so-called duopolies (see e.g. [46] and [49])). When one observes the orbit $\left(x_{n}\right)$ of a point $x \in I$, it is easy to note that the subsequences $\left(x_{p n}\right),\left(x_{p n+1}\right), \ldots,\left(x_{p n+p-1}\right)$ are respectively the orbits of the initial points

$$
x_{0}, \quad f_{0}\left(x_{0}\right), \quad \cdots \quad,\left(f_{p-2} \circ \ldots \circ f_{0}\right)\left(x_{0}\right)
$$

under the iteration of the individual maps

$$
F_{0}, \quad F_{1}, \quad F_{2}, \quad \ldots \quad, F_{p-1},
$$

where $F_{j}=f_{(p-1+j) \bmod p} \circ \ldots \circ f_{j+1} \circ f_{j}$ for $j=0,1, \ldots p-1$. Therefore, one might expect that the dynamics can be completely given by the dynamics of the above individual maps. Indeed, this is true for some characteristics of the dynamical system. For instance, the topological entropy of $\left[f_{0}, \ldots, f_{p-1}\right]$ can be computed by means of the topological entropy of $f_{p-1} \circ \ldots \circ f_{0}$ (see [42]). On the other hand, the $\omega$-limit set $\omega\left(x,\left[f_{0}, \ldots, f_{p-1}\right]\right)$, which is the set of limit points of the orbit with initial condition $x$, can be obtained from the equality

$$
\begin{aligned}
\omega\left(x,\left[f_{0}, \ldots, f_{p}\right]\right) & =\omega\left(x, f_{p-1} \circ \ldots \circ f_{0}\right) \cup \omega\left(f_{0}(x), f_{0} \circ f_{p-1} \circ \ldots \circ f_{1}\right) \cup \ldots \\
& \ldots \cup \omega\left(\left(f_{p-2} \circ \ldots \circ f_{0}\right)(x), f_{p-2} \circ \ldots \circ f_{0} \circ f_{p-1}\right) \\
& =\omega\left(x, F_{0}\right) \cup \omega\left(f_{0}(x), F_{1}\right) \cup \ldots \cup \omega\left(\left(f_{p-2} \circ \ldots \circ f_{0}\right)(x), F_{p-1}\right)
\end{aligned}
$$

where each $\omega\left(z_{j}, F_{j}\right)$ is meant the set of limit points of the orbit of $z_{j}=\left(f_{j-1} \circ \ldots \circ\right.$ $\left.f_{0}\right)(x)$ under the interval map $F_{j}, j=0,1, \ldots, p-1$, (here, $z_{0}=x$ ).

The aim of this paper is to show that, even when many dynamical properties can be studied by the folded dynamical systems, there are several open problems that deserve investigation. The paper is organized in sections and each section covers a topic that includes some proposed open problems. In Section 2 we deal with the set of periods $\operatorname{Per}\left[f_{0}, \ldots, f_{p-1}\right]$ of periodic non-autonomous systems. In Section 3 we 
analyze how this set can be altered by the effect of folding some maps of the periodic non-autonomous system in order to obtain a new system, of possibly shorter period. After this, we present in Section 4, the question of studying the resulting period when we combine strings of two given periodic sequences. Another problem related with periodic orbits appears in Section 5: it is an open problem to determine whether or not the intersection of the sets of periodic points of two commuting interval maps is empty. Section 6 is devoted to the Parrondo's paradox. Finally, we present some interesting applications related to the dynamics of population models described by periodic non-autonomous systems.

\section{Periodic orbits in periodic non-autonomous systems}

When Eq. (1.1) is composed of one map, say $f$, an orbit $\operatorname{Orb}(x, f)=\left(x_{n}\right)$ is said to be periodic if there is $q \in \mathbb{N}:=\{1,2, \ldots\}$ such that $x_{n+q}=x_{n}$ for all $n \geq 0$. The smallest number $q$ satisfying this condition is called the period or order, and denoted by $\operatorname{ord}_{f}(x)$. In the case of discrete dynamical systems on the interval $I$, the wellknown Sharkovsky's theorem characterizes the set of periods of $f$, denoted $\operatorname{Per}(f)$. More precisely, we consider the following order in the set of natural numbers $\mathbb{N}$.

$$
\begin{aligned}
& 3>_{\mathrm{s}} 5>_{\mathrm{s}} 7>_{\mathrm{s}} \ldots>_{\mathrm{s}} 2 \cdot 3>_{\mathrm{s}} 2 \cdot 5>_{\mathrm{s}} 2 \cdot 7>_{\mathrm{s}} \ldots \\
& 2^{n} \cdot 3>_{\mathrm{s}} 2^{n} \cdot 5>_{\mathrm{s}} 2^{n} \cdot 7>_{\mathrm{s}} \ldots>_{\mathrm{s}} 2^{n+1}>_{\mathrm{s}} 2^{n}>_{\mathrm{s}} \ldots>_{\mathrm{s}} 2>_{\mathrm{s}} 1 .
\end{aligned}
$$

For $n \in \mathbb{N} \cup\left\{2^{\infty}\right\}$, define $\mathcal{S}(n)=\left\{m \in \mathbb{N}: n>_{\mathrm{s}} m\right\} \cup\{n\}$ and $\mathcal{S}\left(2^{\infty}\right)=\left\{2^{n}\right.$ : $n \in \mathbb{N} \cup\{0\}\}$. Sharkovsky's theorem states that if $f$ has a periodic orbit (periodic sequence) of period $n$, then it has periodic points (periodic sequences) of period $m \in S(n)$. Moreover, for any $n \in \mathbb{N} \cup\left\{2^{\infty}\right\}$ there is $f \in C(I)$ such that $\operatorname{Per}(f)=\mathcal{S}(n)$ (see [54] or [33] for a recent proof of Sharkovsky's theorem).

In the case of periodic non-autonomous systems, a generalization of Sharkovsky's theorem is given in [11]. For a fixed $p \in \mathbb{N}$, consider a $p$-periodic system defined by the maps $\left[f_{0}, \ldots, f_{p-1}\right]$. For $q \in \mathbb{N}$, define the clusters

$$
\mathcal{A}_{p, q}=\{n: \operatorname{lcm}(n, p)=q \cdot p\}=\left\{n: q=\frac{n}{\operatorname{gcd}(n, p)}\right\} .
$$

Notice that $p \cdot q \in \mathcal{A}_{p, q}$. Now, define the equivalence relation " $\sim p$ " on $\mathbb{N}$ by stating that $n \sim_{p} m, n, m \in \mathbb{N}$, if and only if $n$ and $m$ belong to the same set $\mathcal{A}_{p, q}$ for some $q \in \mathbb{N}$. If we denote any equivalence class $\mathcal{A}_{p, q}$ by $[q]$, we define the order on $\mathbb{N} / \sim_{p}$ by $[n]>_{\mathrm{s}}[m]$ if and only if $n>_{\mathrm{s}} m$. Now, $[q] \in \operatorname{Per}\left(\left[f_{0}, \ldots, f_{p-1}\right]\right) / \sim_{p}$ denotes that $\mathcal{A}_{p, q} \cap \operatorname{Per}\left(\left[f_{0}, \ldots, f_{p-1}\right]\right)$ is nonempty. The generalization in [11] shows if $[n] \in \operatorname{Per}\left(\left[f_{0}, \ldots, f_{p-1}\right]\right) / \sim_{p}$, then for any $[m] \in \mathbb{N} / \sim_{p}$ such that $[n]>_{\mathrm{S}}[m]$, we have $[m] \in \operatorname{Per}\left(\left[f_{0}, \ldots, f_{p-1}\right]\right) / \sim_{p}$. The proof of this result is based on two facts: 
Sharkovsky's theorem and the fact that if $m \in \operatorname{Per}\left(\left[f_{0}, \ldots, f_{p-1}\right]\right)$ and $m \in[q]$, then $q \in \operatorname{Per}\left(f_{p-1} \circ \ldots \circ f_{0}\right)$.

When $p=2$, another approach was used in [24] to characterize the structure of the set of periods $\operatorname{Per}\left(\left[f_{0}, f_{1}\right]\right)$. More precisely, set

$$
\mathbb{N}^{*}:=\mathbb{N} \backslash(\{2 n-1: n \in \mathbb{N}\} \cup\{2\}) .
$$

The following result is given in [24].

Theorem 2.1. Each of the following holds true for a 2-periodic system:

(a) If $\left[f_{0}, f_{1}\right]$ has a periodic orbit of period $n \in \mathbb{N}^{*} \cup\left\{2^{\infty}\right\}$, then $\mathcal{S}(n) \backslash\{1,2\} \subset$ $\operatorname{Per}\left[f_{0}, f_{1}\right]$.

(b) If $2 n+1 \in \operatorname{Per}\left[f_{0}, f_{1}\right], n \geq 1$, then $\mathcal{S}(2 \cdot 3) \backslash\{1\} \subset \operatorname{Per}\left[f_{0}, f_{1}\right]$.

(c) There is a 2-periodic system $\left[f_{0}, f_{1}\right]$ such that $\operatorname{Per}\left(\left[f_{0}, f_{1}\right]\right)$ is $\{1\},\{2\}$ or $\{1,2\}$.

(d) For any $n \in \mathbb{N}^{*} \cup\left\{2^{\infty}\right\}$ : There is a 2-periodic system $\left[f_{0}, f_{1}\right]$ such that one of the following is satisfied.

d.1. $\operatorname{Per}\left(\left[f_{0}, f_{1}\right]\right)=\mathcal{S}(n)$.

d.2. $\operatorname{Per}\left(\left[f_{0}, f_{1}\right]\right)=\mathcal{S}(n) \backslash\{1\}$.

d.3. $\operatorname{Per}\left(\left[f_{0}, f_{1}\right]\right)=\mathcal{S}(n) \backslash\{2\}$.

(e) For any subset of odd numbers $\operatorname{Imp} \subseteq\{2 n+1: n \in \mathbb{N}\}$ there is a 2-periodic system $\left[f_{0}, f_{1}\right]$ such that one of the following is satisfied.

e.1. $\operatorname{Per}\left(\left[f_{0}, f_{1}\right]\right)=\operatorname{Imp} \cup(\mathcal{S}(2 \cdot 3) \backslash\{1\})$.

e.2. $\operatorname{Per}\left(\left[f_{0}, f_{1}\right]\right)=\operatorname{Imp} \cup \mathcal{S}(2 \cdot 3)$.

Notice that the case $\operatorname{Per}\left(\left[f_{0}, f_{1}\right]\right)=\operatorname{Imp} \cup(\mathcal{S}(2 \cdot 3) \backslash\{2\})$ is not allowed, that is, if $2 n+1 \in \operatorname{Per}\left(\left[f_{0}, f_{1}\right]\right)$ for some $n \in \mathbb{N}$, then automatically $2 \in \operatorname{Per}\left(\left[f_{0}, f_{1}\right]\right)$. In addition, for $n \in \mathbb{N}^{*} \cup\left\{2^{\infty}\right\}, n \neq 2 \cdot 3$, there are no continuous maps $f_{0}, f_{1} \in C(I)$ such that $\operatorname{Per}\left(\left[f_{0}, f_{1}\right]\right)=\operatorname{Imp} \cup(\mathcal{S}(n) \backslash\{1\})$ or $\operatorname{Per}\left(\left[f_{0}, f_{1}\right]\right)=\operatorname{Imp} \cup(\mathcal{S}(n) \backslash\{2\})$ or $\operatorname{Per}\left(\left[f_{0}, f_{1}\right]\right)=\operatorname{Imp} \cup \mathcal{S}(n)$.

The following frame summarizes the forcing (where $n>_{2} m$ is meant that the presence of a period $n$ in the alternated system forces the existence of periodic sequences having order $m)$ :

$$
\begin{aligned}
& \{2 \cdot n+1: n \in \mathbb{N}\}>_{2} 2 \cdot 3>_{2} 2 \cdot 5>_{2} 2 \cdot 7>_{2} \ldots \\
& 2^{n} \cdot 3>_{2} 2^{n} \cdot 5>_{2} 2^{n} \cdot 7>_{2} \ldots>_{2} 2^{n}>_{2} \ldots>_{2} 2^{2}>_{2}(1 \text { or } / \text { and } 2) .
\end{aligned}
$$

The generalization of Sharkovsky's theorem given by AlSharawi et al. in [11] lacks the details about the forcing relationship within each equivalence class $[q]=\mathcal{A}_{p, q}$. On the other hand, the result of Cánovas and Linero in [24] gives the exact forcing 
between cycles when $p=2$, but lacks the generality for $p>2$. Since the results in [11] and [22], several attempts have been made to give the exact forcing within each equivalence class $[q][6,8,15]$. Although progress has been made in special cases, the general case is still open, which motivates our first open problem.

Open Problem 2.1. Extend Theorem 2.1 to periodic sequences of maps of arbitrary period, i.e., characterize the set of periods $\operatorname{Per}\left(\left[f_{0}, \ldots, f_{p-1}\right]\right)$ for any positive integer $p$.

In each equivalence class or cluster $[q]=\mathcal{A}_{p, q}$, there is one period, namely $p q$, that does not depend on the intersection between the maps $f_{0}, f_{1}, \ldots, f_{p-1}$, while the other periods need certain intersections between the maps $[8,15]$. This observation leads to dividing the periods into the ones that are generic properties of the intersections, and the ones that are generic properties of the iterations. Indeed, establishing a connection between those two sets was one of the objectives in $[8,6,12]$. Examples were constructed [6] to show that the existence of $m \in \operatorname{Per}\left(\left[f_{0}, \ldots, f_{p-1}\right]\right)$ for some $m \in \mathcal{A}_{p, q}$, where $q>1$ is a power of 2 , does not guarantee that $p q \in \operatorname{Per}\left(\left[f_{0}, \ldots, f_{p-1}\right]\right)$. However, the following problem is still open.

Open Problem 2.2. Suppose $m \in \operatorname{Per}\left(\left[f_{0}, \ldots, f_{p-1}\right]\right)$ for some $m \in \mathcal{A}_{p, q}$, where $q>1$ is an odd number. Prove that $p q \in \operatorname{Per}\left(\left[f_{0}, \ldots, f_{p-1}\right]\right)$.

It is well known that Sharkovsky's theorem works under certain modifications for other one-dimensional spaces, as the circle -with some modifications as a consequence of the degree of a circle map-, even for classes of $n$-dimensional continuous maps, for instance the so-called triangular maps $G\left(x_{1}, x_{2}, \ldots, x_{n}\right)=\left(g_{1}\left(x_{1}\right), g_{2}\left(x_{1}, x_{2}\right), \ldots, g_{n}\left(x_{1}, x_{2}, \ldots, x_{n}\right)\right)$ (see [5] for more details). To this respect:

Open Problem 2.3. Extend Theorem 2.1 to periodic sequences of two continuous circle maps, that is, characterize the set of periods $\operatorname{Per}\left(\left[f_{0}, f_{1}\right]\right)$, where $f_{0}, f_{1}: \mathbb{S}^{1} \rightarrow \mathbb{S}^{1}$ are continuous.

\section{Folding in periodic systems}

For a $p$-periodic system $\left[f_{0}, \ldots, f_{p-1}\right]$, it is possible to fold some of the maps to obtain a system of possibly shorter period. For instance, an obvious situation is the $p$-fold map $F_{0}:=f_{p-1} \circ f_{p-2} \circ \cdots \circ f_{0}$, which changes the $p$-periodic non-autonomous system into an autonomous system. In general, for any $1 \leq k \leq p-1$, we can fold the maps

$$
f_{k-1} \circ \cdots \circ f_{0}=: F_{0}, f_{2 k-1} \circ \cdots \circ f_{k}=: F_{1}, f_{3 k-1} \circ \cdots \circ f_{2 k}=: F_{2}, \cdots
$$

to form another periodic system $\left[F_{0}, F_{1}, \ldots, F_{\frac{p}{\operatorname{gcd}(p, k)}}\right]$. Some caution must be made here about the period of the new periodic system. It may not be $\frac{p}{\operatorname{gcd}(p, k)}$. Indeed, 
take the 4-periodic system $\left[f, f^{-1}, f^{-1}, f\right]$ and $k=2$, then the folded system $\left[F_{0}, F_{1}\right]$ is 1-periodic rather than 2-periodic. The notion of folding was introduced in [6]. The scenario of having $\left[F_{0}, F_{1}, \ldots, F_{\frac{p}{\operatorname{gcd}(p, k)}}\right]$ with a period less than $\frac{p}{\operatorname{gcd}(p, k)}$ is called degenerate scenario and has been avoided. The case when $k$ is a divisor of $p$ is studied in [12] and connections between the cycles of the folded and unfolded systems have been established. However, for general $k$, the connection between the folded and unfolded systems still open for further investigation, which motives the next open problem.

Open Problem 3.1. Consider the p-periodic system $\left[f_{0}, \ldots, f_{p-1}\right]$, and let $1 \leq k \leq p$. Consider the maps $F_{0}, F_{1}, \ldots, F_{\frac{p}{\operatorname{gcd}(p, k)}}$ as defined in (3.1). What is the relationship

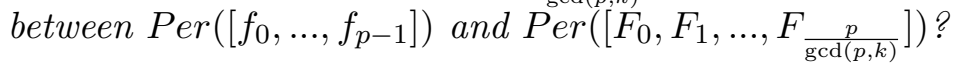

Periodic difference equations with delay of the form $y_{n+1}=g_{n \bmod p}\left(y_{n-(k-1)}\right)$ have been studied by AlSharawi et al. in [10], and a characterization of the periodic structures were given. To visualize the orbits in this case, consider $p=6$ and $k=4$, then orbits of $y_{n+1}=g_{n \text { mod } 6}\left(y_{n-3}\right)$ can be written in matrix form as

$\begin{array}{cccc}y_{-3} & y_{-2} & y_{-1} & y_{0} \\ g_{0} & g_{1} & g_{2} & g_{3} \\ g_{4} & g_{5} & g_{0} & g_{1} \\ g_{2} & g_{3} & g_{4} & g_{5}\end{array}$

From the columns of this matrix, observe that each column gives a periodic system of period $\frac{p}{\operatorname{gcd}(p, k)}$, which can be assumed to be the minimal period. This observation motivates the following problem.

Open Problem 3.2. Suppose there is a p-periodic system $\left[f_{0}, \ldots, f_{p-1}\right]$, which is unknown to us, but we know one of its folded systems $\left[F_{0}, F_{1}, \ldots, F_{\frac{p}{\operatorname{gcd}(p, k)}}\right]$. What kind of similarity (if any) in periodic structure do we have between the unfolded $p$ periodic system $\left[f_{0}, \ldots, f_{p-1}\right]$ and the periodic system with delay $x_{n+1}=F_{n}\left(x_{n-(k-1)}\right)$ ?

\section{Merging periodic sequences}

In the unfolding process of a $\frac{p}{k}$-periodic system $\left[F_{0}, F_{1}, \ldots, F_{\frac{p}{k}}\right]$, we find ourselves dealing with sequences that are merged in a certain way [12]. Therefore, we find the notion of merging two periodic sequences to be related to the topic of the previous section, and therefore, we find it is worth addressing here. Suppose we have two periodic sequences $\left\{a_{n}\right\}$ and $\left\{b_{n}\right\}$ of periods $q_{1}$ and $q_{2}$, respectively. The two sequences can be thought of as two periodic signals or codes coming out of two machines. After each string of length $k_{1}$ produced by the first machine (a $k_{1}$ string of $\left\{a_{n}\right\}$ ), the second machine releases a $k_{2}$ string (a $k_{2}$ string of $\left\{b_{n}\right\}$ ). The obtained signal has the 
structure $\left[a_{n}, b_{n}\right]:=$

$$
\overbrace{a_{1}, a_{2}, \ldots, a_{k_{1}}}^{k_{1} \text { string }}, \overbrace{b_{1}, b_{2}, \ldots, b_{k_{2}}}^{k_{2} \text { string }}, \overbrace{a_{k_{1}+1}, a_{k_{1}+2}, \ldots, a_{2 k_{1}}}^{k_{1} \text { string }}, b_{k_{2}+1}, \ldots
$$

Before we proceed, we clarify the notion by an illustrative example.

Example 4.1. For $n \in \mathbb{N}$, consider $a_{n}=n \bmod 4$ and $b_{n}=4+(n \bmod 6)$. Thus, $\left\{a_{n}\right\}$ is periodic of period $q_{1}:=4$ and $\left\{b_{n}\right\}$ is periodic of period $q_{2}:=6$.

(i) If $k_{1}=2$ and $k_{2}=3$, then

$$
\left[a_{n}, b_{n}\right]=\{0,1,4,5,6,2,3,7,8,9,0,1,4,5,6, \cdots\},
$$

and the period of the formed sequence is 10 .

(ii) If, $k_{1}=2$ and $k_{2}=4$, then

$$
\begin{aligned}
{\left[a_{n}, b_{n}\right]=} & \{0,1,4,5,6,7,2,3,8,9,4,5,0,1,6,7,8,9, \\
& 2,3,4,5,6,7,0,1,8,9,4,5,2,3,6,7,8,9,0,1 \cdots\}
\end{aligned}
$$

and the period of the formed sequence is 36 .

For a better understanding of the structure of the formed sequence $\left[a_{n}, b_{n}\right]$, we write its elements in matrix form as follows:

$\begin{array}{llllllll}a_{0} & a_{1} & \ldots & a_{k_{1}-1} & b_{0} & b_{1} & \ldots & b_{k_{2}-1} \\ a_{k_{1}} & a_{k_{1}+1} & \ldots & a_{2 k_{1}-1} & b_{k_{2}} & b_{k_{2}+1} & \ldots & b_{2 k_{2}-1} \\ a_{2 k_{1}} & a_{2 k_{1}+1} & \ldots & a_{3 k_{1}-1} & b_{2 k_{2}} & b_{2 k_{2}+1} & \ldots & b_{3 k_{2}-1} \\ a_{3 k_{1}} & a_{3 k_{1}+1} & \ldots & a_{4 k_{1}-1} & b_{3 k_{2}} & b_{3 k_{2}+1} & \ldots & b_{4 k_{2}-1} \\ \vdots & \vdots & \ldots & \vdots & \vdots & \vdots & \ldots & \vdots\end{array}$

Now, reading this matrix row by row from left to right gives the formed sequence $\left[a_{n}, b_{n}\right]$. It is obvious that we obtain a periodic sequence in each column. In some cases, it is easy to deduce the new period of the sequence obtained from the merging process, for instance when the merged sequences are disjoint (we establish here the result without proof):

Lemma 4.1. For $n \in \mathbb{N}$, let $\left\{a_{n}\right\}$ and $\left\{b_{n}\right\}$ be two disjoint periodic sequences of periods $q_{1}$ and $q_{2}$, respectively. Also, let $1 \leq k_{1} \leq q_{1}$ and $1 \leq k_{2} \leq q_{2}$. The period of the sequence $\left[a_{n}, b_{n}\right]$ formed in (4.1) is of minimal period $k q$, where $k=k_{1}+k_{2}$ and $q=\operatorname{lcm}\left(\frac{q_{1}}{\operatorname{gcd}\left(k_{1}, q_{1}\right)}, \frac{q_{2}}{\operatorname{gcd}\left(k_{2}, q_{2}\right)}\right)$.

The condition to have the two sequences disjoint is a luxury that one may not have, which leads us to state the following general problem:

Open Problem 4.1. For $n \in \mathbb{N}$, let $\left\{a_{n}\right\}$ and $\left\{b_{n}\right\}$ be two periodic sequences of periods $q_{1}$ and $q_{2}$, respectively. Find the minimal period of the sequence $\left[a_{n}, b_{n}\right]$ formed in (4.1). 


\section{Commuting maps and the problem of shar- ing periodic orbits}

Denote by $\operatorname{Fix}(f)$ and $\mathrm{P}(f)$ the set of fixed and periodic points of a map $f \in C(I)$, respectively. We consider two maps $f_{0}, f_{1} \in C(I)$ such that they commute, that is, $f_{0} \circ f_{1}=f_{1} \circ f_{0}$.

In the fifties of the 20-th century, some authors posed independently the problem of proving whether two commuting continuous interval maps share fixed points. The problem is answered in affirmative for polynomials, as J. F. Ritt pointed out in [51]. Other cases, under restrictive conditions, have also a positive answer (for instance, we mention [32] or [55]). The question on whether $\operatorname{Fix}\left(f_{0}\right) \cap \operatorname{Fix}\left(f_{1}\right)$ is nonempty, that is, $f_{0}$ and $f_{1}$ have a common fixed point, was open for a long time [39]. Finally, Boyce ([21]) and Huneke ([38]) found simultaneously counterexamples which show that in general the answer is negative, there exist two continuous commuting interval maps which do not share any fixed point. The counterexamples constructed in [21] and [38] are given by two maps $f_{0}$ and $f_{1}$ that share periodic points.

Since then, the research on this topic was concentrated in several directions. For instance, to extend the problem to other compact metric spaces or to particular classes of continuous maps ([41], [35], [43]). The problem has been also posed in terms of sharing periodic points which are not necessarily fixed points (see [3] and [57]). Then, it can be expected to raise the following question:

Open Problem 5.1. Is it true that $\mathrm{P}\left(f_{0}\right) \cap \mathrm{P}\left(f_{1}\right) \neq \emptyset$ for commuting continuous interval maps $f_{0}, f_{1} \in C(I)$ ?

Fixed and periodic points are the strongest type of recurrence in dynamical systems. There are weaker notions of recurrence that contain the sets of fixed and periodic points. Namely, a point $x \in X$ is called recurrent if for any open neighborhood $U$ of $x$ there is an increasing sequence $\left\{n_{i}\right\}_{i=1}^{\infty}$ such that $f^{n_{i}}(x) \in U$. If the sequence $\left\{n_{i}\right\}_{i=1}^{\infty}$ has bounded gaps, the point is called uniformly recurrent. If $n_{i}=k i$ for some $k \in \mathbb{N}$ the point is called almost periodic. Denote by $\operatorname{Rec}(f), \operatorname{UR}(f)$ and $\operatorname{AP}(f)$ the sets of recurrent, uniformly recurrent and almost periodic points. It is clear from the definitions that

$$
\operatorname{Fix}(f) \subseteq \mathrm{P}(f) \subseteq \operatorname{AP}(f) \subseteq \mathrm{UR}(f) \subseteq \operatorname{Rec}(f) .
$$

Following the Sharkovsky's order of natural numbers, let $\mathcal{T}_{1}=\{f \in C(I): \mathrm{P}(f)$ is closed $\}, \mathcal{T}_{2}=\left\{f \in C(I): f\right.$ has periodic points of period $\left.2^{n}, n \geq 0\right\}$ and $\mathcal{T}_{3}=\{f \in$ $C(I): f$ has a periodic point which is not a power of two $\}$. The next result was proved in [23].

Theorem 5.1. Assume $f_{0}, f_{1} \in C(I)$ commute. Then 
(a) If $f_{0} \in \mathcal{T}_{1}$, then $\operatorname{Fix}\left(f_{0}\right) \cap \mathrm{P}\left(f_{1}\right) \neq \emptyset$.

(b) If $f_{0} \in \mathcal{T}_{2}$, then $\operatorname{Fix}\left(f_{0}\right) \cap \operatorname{AP}\left(f_{1}\right) \neq \emptyset$.

(c) If $f_{0} \in \mathcal{T}_{3}$, then $\operatorname{Fix}\left(f_{0}\right) \cap \operatorname{UR}\left(f_{1}\right) \neq \emptyset$.

The above result proves Open Problem 5.1 for maps which are simple from the point of view of dynamics. Note that maps of type $\mathcal{T}_{3}$ have positive topological entropy, and therefore, they are chaotic in the sense of Li and Yorke. Chaotic maps in the sense of $\mathrm{Li}$ and Yorke may also exist in the family $\mathcal{T}_{2}$, but they cannot be found in $\mathcal{T}_{1}$, which contains the set of continuous interval maps with finite set of periods (see [18] or [23]). So, if one has to look for counterexamples for Open Problem 5.1, he/she should construct maps having both infinitely many periodic points. In addition, they cannot have a finite number of monotonicity pieces (see [21] and [38]).

We finish this section by a problem that links Theorem 2.1 and Open problem 5.1 .

Open Problem 5.2. Find $\operatorname{Per}\left(\left[f_{0}, f_{1}\right]\right)$ for commuting maps $f_{0}, f_{1} \in C(I)$. More precisely, can the examples of Theorem 2.1 be constructed such that $f_{0}$ and $f_{1}$ commute?

\section{The Parrondo's paradox}

Parrondo's paradox [36] has become an active area of research in many applied sciences like Physics [48], Economy [58] or Biomathematics [59]. As a first approach, we can say that it appears when we alternate different games in a stochastic or deterministic way. Parrondo's paradox exists when the behavior of individual systems and the combined one are completely different. For discrete dynamical systems, the paradox was formulated in [4] by showing that the phenomenon "chaos+chaos=order" and "order+order=chaos" are possible when considering periodic combinations of 1-dimensional quadratic maps. Similar results have been obtained by Boyarsky and collaborators in the random combination of piecewise smooth maps [20]. On the other hand, it was shown in [22] that in some particular cases the paradox is not possible.

The dynamic Parrondo's paradox was studied in detail in [25] as follows. For a map $f \in C(I), I=[0,1]$, denote by $\mathcal{D}(f)$ the set of dynamic properties of $f$ (for instance to have positive topological entropy or exhibiting chaos in the sense of $\mathrm{Li}$ and Yorke), and define $\mathcal{D}\left(\left[f_{0}, f_{1}\right]\right)$ similarly. Let $J=[a, b] \subseteq I=[0,1]$ and

denote by $\varphi_{J}: J \rightarrow I$ a linear map such that $\varphi_{J}(a)=0$ and $\varphi_{J}(b)=1$. Define $f_{J}:[0,1] \rightarrow[0,1]$ by $f_{J}(x)=\varphi_{J}^{-1} \circ f \circ \varphi_{J}(x)$ if $x \in J, f_{J}(0)=0, f_{J}(1)=1$, and linear on any connected component of $[0,1] \backslash J$. A dynamic property $P \in \mathcal{D}(f)$ is an L-property if for any continuous map $f$ and any compact subinterval $J \subseteq[0,1]$, it is held that $P \in \mathcal{D}(f) \cap \mathcal{D}\left(f_{J}\right)$. The fact that if $f_{0}, f_{1} \in C(I)$, then the dynamics of the sequence $\left[f_{0}, f_{1}\right]$ is complicated (or simple) if and only if the dynamics of $f_{0} \circ f_{1}$ 
is complicated (or simple), jointly with $\mathrm{L}$-properties are the key for analyzing the Parrondo's paradox as the following result shows [25].

Theorem 6.1. Let $P_{i}, i=1,2,3$, be L-properties. Then there are $f_{0}, f_{1} \in C(I)$ such that $P_{1} \in \mathcal{D}\left(f_{0}\right), P_{2} \in \mathcal{D}\left(f_{1}\right)$ and $P_{3} \in \mathcal{D}\left(f_{0} \circ f_{1}\right)$.

In particular, we can construct maps such that $f_{0}$ and $f_{1}$ have a complicated (simple) L-property and $f_{0} \circ f_{1}$ has not this property. $\left[P \in \mathcal{D}\left(f_{0}\right) \cap \mathcal{D}\left(f_{1}\right)\right.$ and $\left.P \notin \mathcal{D}\left(f_{0} \circ f_{1}\right)\right]$. For instance, we consider the topological entropy (see e.g. [2] or [5] for definition and basic properties of topological entropy), which is a useful tool to decide whether a map has a complicated dynamics. From Theorem 6.1, we can construct two continuous interval maps, $f_{0}$ and $f_{1}$, with zero topological entropy (and hence simple) such that $f_{0} \circ f_{1}$ has positive topological entropy (and therefore a complicated dynamics), because the properties zero topological entropy and positive topological entropy are L-properties. However, we must emphasize that, although Theorem 6.1 shows the existence of paradox in a general way for a very large list of dynamical properties, the constructions made for proving it cannot be done when one consider fixed maps like for instance a member of the logistic family $f_{a}(x)=a x(1-x)$, $1 \leq a \leq 4$ and $x \in[0,1]$.

Open Problem 6.1. State an analogous result to Theorem 6.1 when the maps $f_{0}$ and $f_{1}$ commute.

Consider the well-known logistic family $f_{a}(x)=a x(1-x), a \in[1,4]$. More precisely, we consider two maps $f_{a}$ and $f_{b}$ and wonder about the existence of paradox for parameters $a$ and $b$. In [27], the existence of Parrondo's paradox is shown for several parameters values. However, although $f_{a} \circ f_{b}$ may exhibit the paradox, it is observed that several combinations like $f_{a} \circ f_{b} \circ f_{a}$ do not exhibit the paradox.

Open Problem 6.2. Characterize the set of parameters $a, b \in[1,4]$ such that any combination of maps $f_{a}$ and $f_{b}$ exhibit the Parrondo's paradox. Is this set nonempty?

Denote the topological entropy of a continuous map $f$ by $h(f)$. In [26], numerical simulations show that Parrondo's paradox cannot be exhibited by maps with positive topological entropy, that is, if $\min \left\{h\left(f_{a}\right), h\left(f_{b}\right)\right\}>0$, then numerical simulations show that $h\left(f_{a} \circ f_{b}\right)>0$, and therefore the Parrondo's paradox cannot be exhibited (see Figure 1).

Open Problem 6.3. In the logistic family, prove or disprove that $\min \left\{h\left(f_{a}\right), h\left(f_{b}\right)\right\}>$ 0 implies that $h\left(f_{a} \circ f_{b}\right)>0$.

\section{Applications}

In population dynamics, one dimensional models usually take the form $x_{n+1}=$ $x_{n} f\left(x_{n}\right)$, where $x_{n}$ represents the density of a population at discrete time $n$ and $f(t)$ 

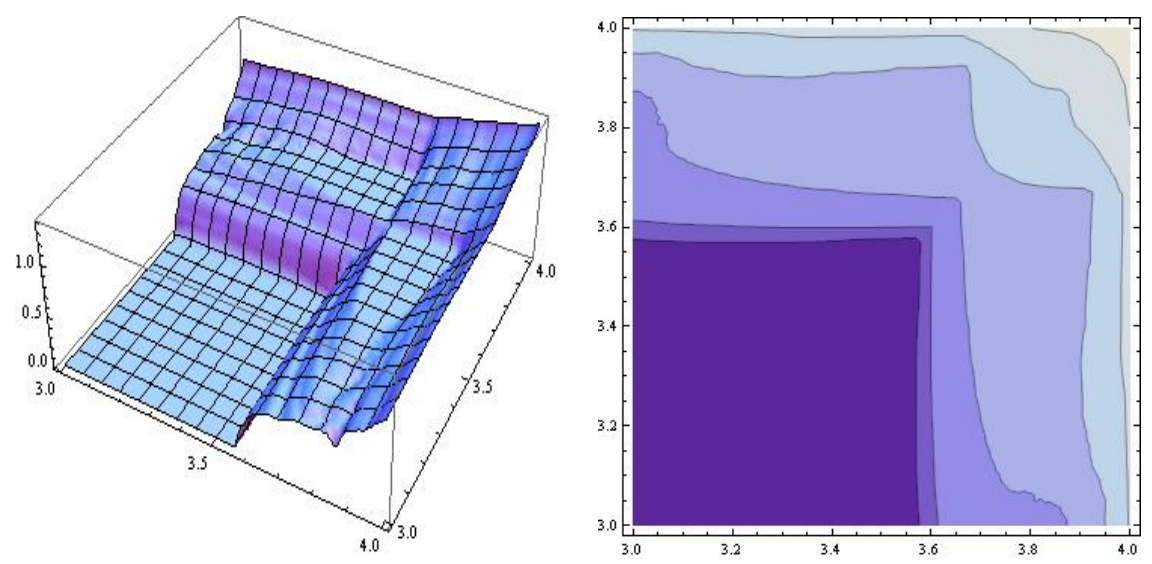

Figure 1: On the left we show the topological entropy of $F$ with accuracy $10^{-3}$. On the right, we present the projection of topological entropy where stronger colors means lower topological entropy. The darkest region represents those parameter values for which the topological entropy is zero up to the prescribed accuracy.

is a function that reflects certain characteristics of the studied species. For instance, $f(t)=\frac{k \mu}{k+(\mu-1) t}, \quad \mu>1$ is used for the Beverton-Holt model [17], $f(t)=a t(b-t)$ is used for the logistic model [47], and $f(t)=b e^{-k t}$ is used for the Ricker model [50]. Forcing periodic harvesting or stocking in a deterministic environment leads to investigating the dynamics of models in the from

$$
x_{n+1}=x_{n} f\left(x_{n}\right) \pm h_{n},
$$

where $\left\{h_{n}\right\}$ is a $p$-periodic sequence that represents harvesting or stocking quotas. See [7] and the references therein for more details and some open questions. We find Problem 3.1 in [7] to be suitable within the context of this paper.

Open Problem 7.1. Consider Eq. (7.1) with stocking (i.e. $+h_{n}$ ) and assume this equation has a global attractor (like when $f(t)=\frac{b t}{1+t}[14]$ ). Let $\left\{\hat{h}_{n}\right\}$ be a permutation of $\left\{h_{n}\right\}$. Define $x_{a v}$ and $\hat{x}_{a v}$ to be the average of the global attractors associated with $\left\{h_{n}\right\}$ and $\left\{\hat{h}_{n}\right\}$, respectively. How does $x_{a v}$ relate to $\hat{x}_{a v}$ ?

Although it is tempting to believe that increasing constant yield harvesting in population models leads to a decline in the population, recent results show otherwise $[56,52]$ and the phenomenon is known as the hydra effect [1]. In fact, this notion lead to a fertile area of research; see for instance [53, 44, 45] and the references therein. When we confine models in Eq. (7.1) to contest-competition models and force the harvesting to be constant yield harvesting (i.e., $h_{n}=h$ for all $n$ ), then the hydra effect is not known to take place. For instance, if we take the Beverton-Holt model with harvesting [13], then the global attractor is decreasing in $h$. However, the effect of periodic harvesting is not characterized yet, which motivates our next problem. 
Open Problem 7.2. Consider Eq. (7.1) with harvesting (i.e. $-h_{n}$ ). Investigate the effect of ordering the elements of the sequence $\left\{h_{n}\right\}$ on the population. On other words, assume that we have a periodic sequence of harvesting quotas but we have the freedom to permute its elements. Which permutation of $\left\{h_{n}\right\}$ plays on the advantage of the population in terms of the basin of attraction and in terms of the arithmetic average of the attractor?

\section{Acknowledgement}

J. S. Cánovas and A. Linero have been partially supported by the grants MTM201123221 from Ministerio de Ciencia e Innovación (Spain) and 08667/PI/08 from Programa de Generación de Conocimiento Científico de Excelencia de la Fundación Séneca, Agencia de Ciencia y Tecnologa de la Comunidad Autónoma de la Región de Murcia (II PCTRM 2007-10).

\section{References}

[1] Abrams, P. A.: When does greater mortality increase population size? The long story and diverse mechanisms underlying the hydra effect, Ecol. Lett. 12, 462-474 (2009).

[2] Adler, R. L., Konheim, A. G., McAndrew, M. H.: Topological entropy, Trans. Amer. Math. Soc. 114, 309-319 (1965).

[3] Alikhani-Koopaei, A.: On common fixed points and periodic points of commuting functions, Internat. J. Math. \& Math. Sci. 21, 269-276 (1998).

[4] Almeida, J., Peralta-Salas, D., Romera, M.: Can two chaotic systems give rise to order?, Phys. D 200, 124-132 (2005).

[5] Alsedá, L., Llibre, J., Misiurewicz, M.: Combinatorial dynamics and entropy in dimension one, World Scientific, Singapore, 1993.

[6] Al-Salman, A., AlSharawi, Z., A new characterization of periodic oscillations in periodic difference equations, Chaos Solitons Fractals, 44, 921-928 (2011).

[7] AlSharawi, Z., Harvesting and stocking in contest competition models: Open problems and conjectures, pre-print.

[8] AlSharawi, Z., Periodic orbits in periodic discrete dynamics, Comput. Math. Appl. 56, 1966-1974 (2008).

[9] AlSharawi, Z., Angelos, J.: On the periodic logistic equation, Appl. Math. Comput. 180, 342-352 (2006). 
[10] AlSharawi, Z., Angelos, J., Elaydi, S.: Existence and stability of periodic orbits of periodic difference equations with delays, Internat. J. Bifur. Chaos Appl. Sci. Engrg. 18, 203-217 (2008).

[11] AlSharawi, Z., Angelos, J., Elaydi, S., Rakesh, L.: An extension of Sharkovsky's theorem to periodic difference equations, J. Math. Anal. Appl. 316, 128-141 (2006).

[12] AlSharawi, Z., Cánovas, J. S., Linero, A.: Folding and unfolding in periodic difference equations, pre-print.

[13] AlSharawi, Z., Rhouma, M.: The Beverton-Holt model with periodic and conditional harvesting, J. Biol. Dyn. 3, 463-478 (2009).

[14] AlSharawi, Z., Rhouma, M.: The discrete Beverton-Holt model with periodic harvesting in a periodically fluctuating environment, Adv. Difference Equ. (2010), Article ID 215875, doi:10.1155/2010/215875.

[15] Alves, J. F.: What we need to find out the periods of a periodic difference equation, J. Difference Equ. Appl. 15, 833-847 (2009).

[16] Alves, J. F., Silva, L.: Periodic paths on nonautonomous graphs, Linear Algebra Appl. 437, 1003-1015 (2012).

[17] Beverton, R. J. H., Holt, S. J.: On the dynamics of exploited fish populations, The Blackburn Press, New Jersey (2004).

[18] Block, L., Coppel, W. A.: Dynamics in one dimension, Lecture Notes in Mathematics 1513, Springer-Verlag, Berlin, 1992.

[19] Block, L., Keesling, J., Li, S., Peterson, K.: An improved algorithm for computing topological entropy, J. Stat. Phys. 55, 929-939 (1989).

[20] Boyarsky, A., Gora, P., Islam, M. S.: Randomly chosen chaotic maps can give rise to nearly ordered behavior, Phys. D 210, 284-294 (2005).

[21] Boyce, W. M.: Commuting functions with no common fixed point, Trans. Amer. Math. Soc. 137, 77-92 (1969).

[22] Cánovas, J. S.: Analyzing when the dynamic Parrondo's paradox is not possible, Internat. J. Bifur. Chaos Appl. Sci. Engrg. 20, 2975-2978 (2010).

[23] Cánovas, J. S., Linero, A.: On the dynamics of composition of commuting interval maps, J. Math. Anal. Appl. 305, 296-303 (2005).

[24] Cánovas, J. S., Linero, A.: Periodic structure of alternating continuous interval maps, J. Difference Equ. Appl. 12, 847-858 (2006).

[25] Cánovas, J. S., Linero, A., Peralta-Salas, D., Dynamic Parrondo's paradox, Phys. D 218, 177-184 (2006). 
[26] Cánovas, J. S., Muñoz, M.: Computing topological entropy for periodic sequences of unimodal maps, pre-print.

[27] Cánovas, J. S., Muñoz, M.: Revisiting Parrondo's paradox for the logistic family, Fluct. Noise Lett. (2013) doi: 10.1142/S0219477513500156.

[28] Cushing, J., Henson, S.: The effect of periodic habitat fluctuations on a nonlinear insect population model, J. Math. Biol. 36, 201-226 (1997).

[29] D'Aniello, E., Oliveira, H.: Pitchfork bifurcation for non-autonomous interval maps, J. Difference Equ. Appl. 15, 291-302 (2009).

[30] D'Aniello, E., Steele, T.H.: Stability in the family of $\omega$-limit sets of alternating systems, J. Math. Anal. Appl. 389, 1191-1203 (2012).

[31] D'Aniello, E., Steele, T.H.: The $\omega$-limit sets of alternating systems, J. Difference Equ. Appl. 17, 1793-1799 (2011).

[32] DeMarr, R.: Common fixed points for commuting contraction mappings, Pacific J. Math. 13, 1139-1141 (1963).

[33] Du, B. S.: A simple proof of Sharkovsky's theorem, Amer. Math. Monthly 111, 595-599 (2004).

[34] Elaydi, S., Sacker, R.: Periodic difference equations, population biology and the Cushing-Henson conjectures, Math. Biosci. 201, 195-207 (2006).

[35] Grinč, M.: On common fixed points of commuting triangular maps, Bull. Polish Acad. Sci. Math. 47, 61-67 (1999).

[36] Harmer, G.P., Abbott, D.: Game theory: Losing strategies can win by Parrondo's paradox, Nature 402, 864 (1999).

[37] Harmer, G.P., Abbott, D.: Parrondo's paradox, Statist. Sci. 14, 206-213 (1999).

[38] Huneke, J. P.: On common fixed points of commuting functions on an interval, Trans. Amer. Math. Soc. 139, 371-381 (1969).

[39] Isbell, J. R.: Commuting mappings of trees, Bull. Amer. Math. Soc. 63, 419 (1957).

[40] Jillson, D. A.: Insect populations respond to fluctuating environments, Nature 288, 699-700 (1980).

[41] Jungck, G.: Common fixed points for commuting and compatible maps on compacta, Proc. Amer. Math. Soc. 103, 977-983 (1988).

[42] Kolyada, S., Snoha, L'.: Topological entropy of nonautononous dynamical systems, Random Comput. Dyn. 4, 205-233 (1996).

[43] Linero, A.: Common fixed points for commuting Cournot maps, Real Anal. Exchange 28, 121-145 (2002). 
[44] Liz, E.: Complex dynamics of survival and extinction in simple population models with harvesting, Theor. Ecol. 3, 209-221 (2010).

[45] Liz, E. Ruiz-Herrera, A.: The hydra effect, bubbles, and chaos in a simple discrete population model with constant effort harvesting, J. Math. Biol. 65, 997-1016 (2012).

[46] Matsumoto, A., Nonaka, Y.: Statistical dynamics in a chaotic Cournot model with complementary goods, Journal of Economic Behavior \& Organization 61, 769-783 (2006).

[47] May, R. M.: Simple mathematical models with very complicated dynamics, Nature 261, 459-467 (1976).

[48] Parrondo, J. M. R., Harmer, G. P., Abbott, D.: New paradoxical games based on Brownian ratchets, Phys. Rev. Lett. 85, 5226-5229 (2000).

[49] Puu, T.: Chaos in duopoly pricing, Chaos Solitons Fractals 1, 573-581 (1991).

[50] Ricker, W. E.: Stock and recruitment, Journal of the Fisheries Research Board of Canada 11, 559-623 (1954).

[51] Ritt, J. F.: Permutable rational functions, Trans. Amer. Math. Soc. 25, 399-448 (1923).

[52] Schreiber, S. J.: Chaos and population disappearances in simple ecological models, J. Math. Biol. 42, 239-260 (2001).

[53] Seno, H.: A paradox in discrete single species population dynamics with harvesting/thinning. Math. Biosci. 214, 63-69 (2008).

[54] Sharkovsky, A.N.: Coexistence of cycles of a continuous transformation of a line into itself, Ukrain. Mat. Zh. 16, 61-71 (1964)(in Russian).

[55] Shields, A. L.: On fixed points of commuting analytic functions, Proc. Amer. Math. Soc. 15, 703-706 (1964).

[56] Sinha, S., Parthasarathy, S.: Unusual dynamics of extinction in a simple ecological model, Proc. Natl. Acad. Sci. USA 93, 1504-1508 (1996).

[57] Steele, T. H.: A note on periodic points and commuting functions, Real Anal. Exchange 24, 781-790 (1998/9).

[58] Spurgin, R., Tamarkin, M.: Switching investments can be a bad idea when Parrondo's paradox applies, J. Behavioral Finance 6, 15-18 (2005).

[59] Wolf, D. M., Vazirani, V. V., Arkin, A. P.: Diversity in times of adversity: probabilistic strategies in microbial survival games, J. Theoret. Biol. 234, 227$253(2005)$. 